\title{
The ortho-nitrophenol (ONPG) test and acid from lactose in Gram-negative genera
}

\author{
S. P. LAPAGE ${ }^{1}$, ANDROUlla efstratiou, AND L. R. Hill \\ From the National Collection of Type Cultures, Central Public Health Laboratory, London
}

SYNOPSIS The results are given of the ortho-nitrophenol test for beta-galactosidase production (ONPG test) on 588 strains of 123 aerobic species of bacteria, representing 30 genera. Apart from some strains of Erwinia herbicola (synonym Chromobacterium typhiflavum) and of Yersinia spp, these strains were not members of the Enterobacteriaceae, in which family the ONPG test is widely used and well documented. The strains were also tested for acid production from 1, 5, and 10\% lactose and the findings are discussed in relation to the ONPG test.

The ortho-nitrophenol test for beta-galactosidase production (ONPG test) has been in widespread use since the value of the test was first reported by Le Minor and Ben Hamida (1962). In lactose-fermenting bacteria the breakdown of lactose to glucose and galactose involves the enzyme beta-galactosidase, but some organisms may give a positive result in the ONPG test yet fail to produce enough acid from lactose to change the indicator under the test conditions. This may be due to lack of permease to allow sufficient lactose to enter the cells, though an adequate number of molecules of beta-galactosidase may diffuse out of the cells to produce the yellow ortho-nitrophenol from the colourless ortho-nitrophenyl-beta-D-galactopyranoside, used as a substrate in the ONPG test. The ONPG test is a much more sensitive test than conventional acid from lactose tests. Moreover, other species can oxidize lactose to lactobionic acid, eg, Acinetobacter anitratus and several pseudomonads (Stodola and Lockwood, 1947; Villecourt and Blachère, 1955; Miller and Von Riesen, 1968). Lactobionic acid production does not involve beta-galactosidase and thus such species are ONPG negative and acid from lactose positive.

Most investigations of the ONPG test and acid from lactose tests have been concerned chiefiy with Enterobacteriaceae (Szturm-Rubinsten and Piéchaud, 1962, 1963; Lapage and Jayaraman, 1964;

${ }^{1}$ Please send any communications to: Dr S. P. Lapage, National Collection of Type Cultures, Central Public Health Laboratory, Colindale Avenue, London, NW9 5HT.

Received for publication 31 July 1973.
Lubin and Ewing, 1964; Giammanco, 1966; Costin 1966). Some data for both tests for non-enterobacterial species, mainly pseudomonads, have been published by Le Minor and Ben Hamida (1962) and Bülow (1964), while Tacquet, Tison, Polspoel, Roos, and Devulder (1966) found the occasional ONPG positive strain among some species of Mycobacterium. In the present work, the range of non-enterobacterial, Gram-negative, aerobic organisms studied in both tests has been extended to $\mathbf{3 0}$ genera representing 123 named species and varieties.

\section{Materials and Methods}

\section{ORGANISMS}

A total of 588 strains have been examined. All, except 30, were NCTC strains and have been routinely checked and characterized by a wide range of tests over the years in the National Collection of Type Cultures. The additional 30 strains were newly isolated strains sent to the NCTC for identification: 10 strains of Eikenella (synonym Bacteroides) corrodens (Hill, Snell, and Lapage, 1970), 10 strains of Pseudomonas cepacia (Snell, Hill, and Lapage, 1972), and 10 strains of $P$. stutzeri (Lapage, Hill, and Reeve, 1968).

The strains examined are listed by species names in table $\mathrm{I}$.

\section{ONPG TEST}

This was carried out by the method of Lowe (1962, method 2, without benzene or toluene treatment). Results were read up to five days' incubation at optimum temperature, usually $37^{\circ} \mathrm{C}$. 


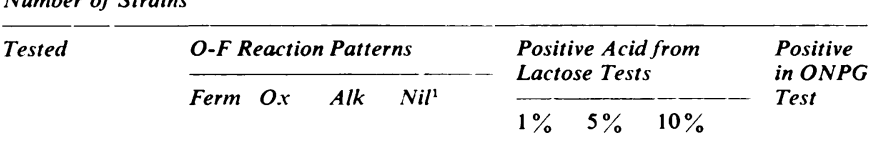

Achromobacter citroalcaligenes

Achromobacter conjunctivae

Achromobacter haemolyticus var. haemolyticus

Achromobacter haemolyticus var. alcaligenes

Achromobacter metalcaligenes

Achromobacter mucosus

Achromobacter xylosoxidans

Acinetobacter anitratus

Acinetobacter /woffii

Actinobacillus actinomycetemcomitans

Actinobacillus equuli

Actinobacillus lignieresi

Actinobacillus spp.

Actinobacillus suis

Aeromonas formicans

Aeromonas liquefaciens

Aeromonas salmonicida

Aeromonas shigelloides

Alcaligenes denitrificans

Alcaligenes faecalis

Alcaligenes odorans

Alcaligenes viscosus

Alysiella filiformis

Bordetella bronchiseptica

Bordetella parapertussis

Bordetella pertussis

Brucella abortus

Brucella melitensis

Brucella neotomae

Brucella ovis

Brucella suis

Campylobacter bubulus

Campylobacter fetus

Cardiobacterium hominis

Chromobacterium amethystinum

Chromobacterium lividum

Comamonas percolans

Cytophaga sp.

Eikenella corrodens

Erwinia herbicola

Flavobacterium meningosepticum

Gemella haemolysans

Haemophilus aphrophilus

Haemophilus canis

Haemophilus gallinarum

Haemophilus haemolyticus

Haemophilus influenzae

Haemophilus parahaemolyticus

Haemophilus parainfluenzae

Haemophilus paraphrohaemolyticus

Haemophilus paraphrophilus

Haemophilus suis

Haemophilus vaginalis

Haemophilus sp.

Moraxella bovis

Moraxella kingii

Moraxella lacunata

Moraxella liquefaciens

Moraxella nonliquefaciens

Moraxella osloensis

Moraxella phenylpyruvica

Moraxella sp.

Moraxella saccharolytica

Neisseria animalis

Neisseria canis

Neisseria catarrhalis

Neisseria caviae

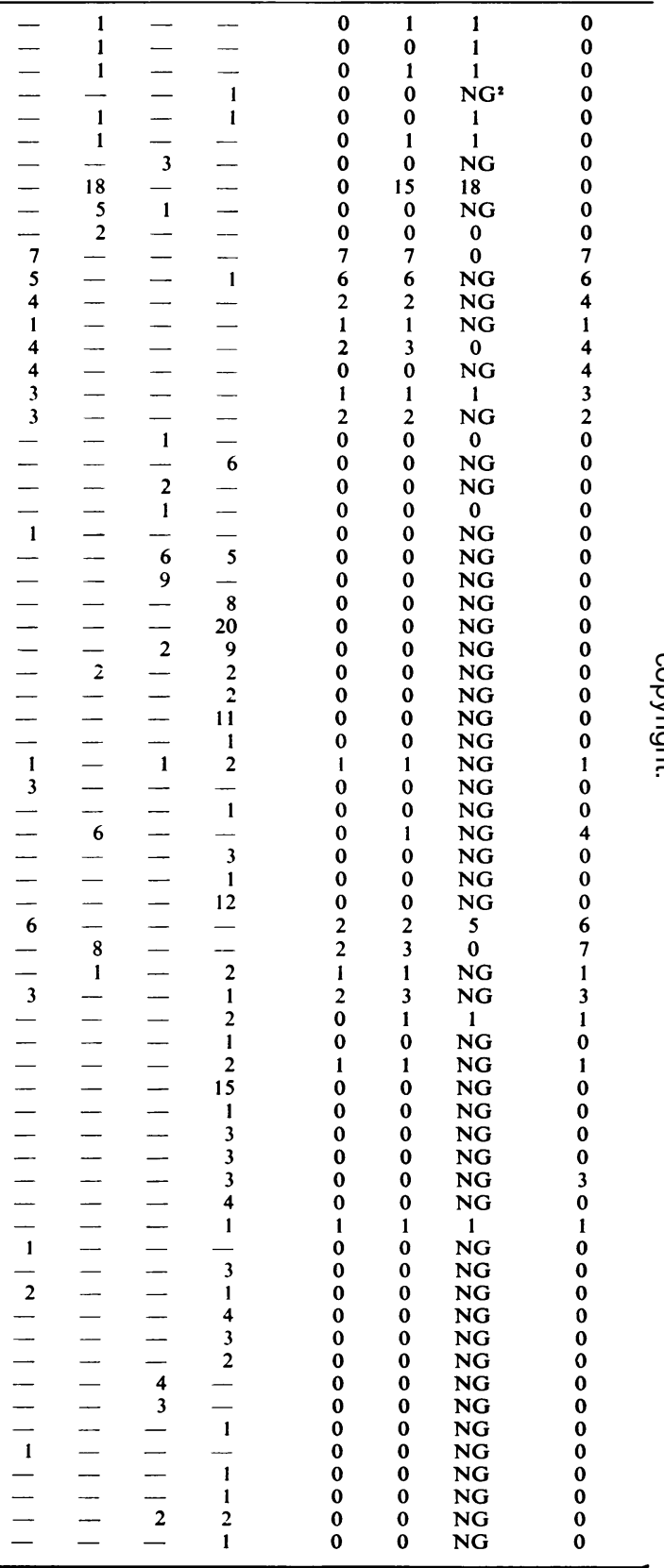

Table I Results of the $O-F$, acid from lactose, and ONPG tests

'Ferm = fermentative; $O x=$ oxidative $;$ Alk = alkali-producing; nil = no reaction. ${ }^{2} \mathrm{~N}$ G $=$ no growth. 


\begin{tabular}{|c|c|c|c|c|c|c|c|c|c|}
\hline \multirow[t]{4}{*}{ Species } & \multicolumn{9}{|c|}{ Number of Strains } \\
\hline & \multirow[t]{3}{*}{ Tested } & \multicolumn{4}{|c|}{ O-F Reaction Patterns } & \multirow{2}{*}{\multicolumn{3}{|c|}{$\begin{array}{l}\text { Positive Acid from } \\
\text { Lactose Tests }\end{array}$}} & \multirow{3}{*}{$\begin{array}{l}\text { Positive } \\
\text { in ONPG } \\
\text { Test }\end{array}$} \\
\hline & & \multirow[t]{2}{*}{ Ferm } & \multirow[t]{2}{*}{$O x$} & \multirow[t]{2}{*}{$A l k$} & \multirow[t]{2}{*}{$N i l^{1}$} & & & & \\
\hline & & & & & & $1 \%$ & $5 \%$ & $10 \%$ & \\
\hline Neisseria cinerea & 1 & - & - & - & 1 & $\mathbf{0}$ & $\mathbf{0}$ & $\mathrm{NG}^{2}$ & $\mathbf{0}$ \\
\hline Neisseria cuniculi & 1 & - & - & - & 1 & 0 & $\mathbf{0}$ & NG & $\mathbf{0}$ \\
\hline Neisseria denitrificans & 1 & 1 & - & - & 一 & $\mathbf{0}$ & $\mathbf{0}$ & NG & $\mathbf{0}$ \\
\hline Neisseria elongata & 1 & - & - & - & 1 & $\mathbf{0}$ & $\mathbf{0}$ & NG & $\mathbf{0}$ \\
\hline Neisseria flavescens & 3 & - & - & - & 3 & $\mathbf{0}$ & $\mathbf{0}$ & NG & 0 \\
\hline Neisseria gonorrhoeae & 11 & - & - & - & 11 & $\mathbf{0}$ & $\mathbf{0}$ & NG & 0 \\
\hline Neisserio lactamicus & 3 & - & 2 & 1 & - & 1 & 3 & NG & 3 \\
\hline Neisseria meningitidis & 10 & - & 3 & - & 7 & $\mathbf{0}$ & $\mathbf{0}$ & NG & $\mathbf{0}$ \\
\hline Neisseria mucosa & 3 & 3 & - & - & - & $\mathbf{0}$ & $\mathbf{0}$ & NG & $\mathbf{0}$ \\
\hline Neisseria pharyngis & 1 & - & 一 & - & 1 & 0 & 0 & NG & 0 \\
\hline Pasteurella bettii & 3 & 3 & - & - & - & $\mathbf{0}$ & 0 & NG & $\mathbf{0}$ \\
\hline Pasteurella haemolytiea & 10 & 10 & - & - & - & 2 & 2 & $\mathbf{N G}$ & 2 \\
\hline Pasteurella haemolytica var. ureae & 9 & 8 & - & - & 1 & $\mathbf{0}$ & $\mathbf{0}$ & NG & $\mathbf{0}$ \\
\hline Pasteurella pneumotropica & 10 & 10 & - & - & - & $\mathbf{0}$ & $\mathbf{0}$ & NG & 4 \\
\hline Pasteurella multocida & 32 & 32 & 一 & - & 一 & 3 & 3 & NG & 3 \\
\hline Pasteurella spp. & 10 & 10 & - & 一 & - & $\mathbf{0}$ & $\mathbf{0}$ & NG & 2 \\
\hline Plesiomonas shigelloides & 6 & 6 & - & - & - & 5 & 5 & 0 & 5 \\
\hline Pseudomonas acidovorans & 2 & - & - & 1 & 1 & $\mathbf{0}$ & $\mathbf{0}$ & $\mathbf{0}$ & $\mathbf{0}$ \\
\hline Pseudomonas aeruginosa & 16 & - & 16 & - & - & $\mathbf{0}$ & $\mathbf{0}$ & 15 & $\mathbf{0}$ \\
\hline Pseudomonas alcaligenes & 2 & - & - & 2 & - & $\mathbf{0}$ & $\mathbf{0}$ & $\mathbf{0}$ & $\mathbf{0}$ \\
\hline Pseudomonas aminovorans & 1 & - & 1 & - & 一 & $\mathbf{0}$ & $\mathbf{0}$ & $\mathbf{0}$ & 1 \\
\hline Pseudomonas atlantica & 1 & - & - & 1 & - & $\mathbf{0}$ & $\mathbf{0}$ & $\mathbf{0}$ & 1 \\
\hline Pseudomonas aureofaciens & 1 & - & 1 & - & - & $\mathbf{0}$ & $\mathbf{0}$ & 1 & $\mathbf{0}$ \\
\hline Pseudomonas cepacia & 14 & 一 & 14 & 一 & - & $\mathbf{0}$ & 3 & 10 & 3 \\
\hline Pseudomonas chlororaphis & 1 & - & 1 & - & - & $\mathbf{0}$ & $\mathbf{0}$ & $\mathbf{0}$ & $\mathbf{0}$ \\
\hline Pseudomonas cruciviae & 1 & 一 & - & 1 & - & $\mathbf{0}$ & $\mathbf{0}$ & $\mathbf{0}$ & $\mathbf{0}$ \\
\hline Pseudomonas denitrificans & 1 & - & 1 & - & - & $\mathbf{0}$ & $\mathbf{0}$ & NG & $\mathbf{0}$ \\
\hline Pseudomonas diminuta & 1 & - & - & 1 & - & 0 & $\mathbf{0}$ & $\mathbf{0}$ & $\mathbf{0}$ \\
\hline Pseudomonas fluorescens & 4 & - & 4 & - & - & $\mathbf{0}$ & $\mathbf{0}$ & 4 & $\mathbf{0}$ \\
\hline Pseudomonas fragi & 1 & - & 1 & - & - & $\mathbf{0}$ & $\mathbf{0}$ & 1 & 0 \\
\hline Pseudomonas graveolens & 2 & - & 2 & - & - & $\mathbf{0}$ & 2 & 2 & $\mathbf{0}$ \\
\hline Pseudomonas lemonnieri & 1 & - & 1 & - & 一 & $\mathbf{0}$ & $\mathbf{0}$ & NG & $\mathbf{0}$ \\
\hline Pseudomonas mallei & 9 & - & - & - & 9 & $\mathbf{0}$ & $\mathbf{0}$ & 8 & $\mathbf{0}$ \\
\hline Pseudomonas maltophilia & 7 & - & 7 & - & - & $\mathbf{0}$ & $\mathbf{0}$ & $\mathbf{0}$ & 0 \\
\hline Pseudomonas mucidolens & 1 & - & 1 & - & - & $\mathbf{0}$ & 1 & 1 & $\mathbf{0}$ \\
\hline Pseudomonas nigrifaciens & 1 & - & 1 & - & - & $\mathbf{0}$ & $\mathbf{0}$ & 0 & 1 \\
\hline Pseudomonas oleovorans & 1 & - & 1 & - & - & $\mathbf{0}$ & $\mathbf{0}$ & NG & $\mathbf{0}$ \\
\hline Pseudomonas ovalis & 2 & - & 2 & - & - & $\mathbf{0}$ & $\mathbf{0}$ & 2 & $\mathbf{0}$ \\
\hline Pseudomonas pavonacea & 1 & - & - & 1 & - & $\mathbf{0}$ & $\mathbf{0}$ & $\mathbf{0}$ & $\mathbf{0}$ \\
\hline Pseudomonas pseudomallei & 13 & - & 13 & - & - & 1 & 11 & 13 & $\mathbf{0}$ \\
\hline Pseudomonas putrefaciens & 4 & 4 & - & - & - & $\mathbf{0}$ & $\mathbf{0}$ & 0 & $\mathbf{0}$ \\
\hline Pseudomonas rubescens & 1 & - & - & 1 & - & $\mathbf{0}$ & $\mathbf{0}$ & $\mathbf{0}$ & $\mathbf{0}$ \\
\hline Pseudomonas stutzeri & 13 & - & 13 & - & 一 & $\mathbf{0}$ & $\mathbf{0}$ & 2 & $\mathbf{0}$ \\
\hline Pseudomonas syncyanea & 1 & - & 1 & - & 一 & $\mathbf{0}$ & $\mathbf{0}$ & NG & 0 \\
\hline Pseudomonas synxantha & 1 & 一 & 1 & - & - & $\mathbf{0}$ & $\mathbf{0}$ & 1 & $\mathbf{0}$ \\
\hline Pseudomonas testosteroni & 1 & - & - & 1 & - & $\mathbf{0}$ & $\mathbf{0}$ & 0 & $\mathbf{0}$ \\
\hline Pseudomonas spp. achromogenic & 5 & - & 5 & - & - & $\mathbf{0}$ & $\mathbf{0}$ & 1 & $\mathbf{0}$ \\
\hline Ramibacterium pseudoramosum & 1 & - & - & - & 1 & $\mathbf{0}$ & $\mathbf{0}$ & NG & $\mathbf{0}$ \\
\hline Simonsiella crassa & 1 & 1 & - & 一 & - & 0 & $\mathbf{0}$ & $\mathbf{N G}$ & $\mathbf{0}$ \\
\hline Spirillum serpens & 1 & - & - & - & 1 & 0 & $\mathbf{0}$ & NG & $\mathbf{0}$ \\
\hline Streptobacillus moniliformis & 1 & - & - & 1 & - & $\mathbf{0}$ & 0 & NG & $\mathbf{0}$ \\
\hline Vibrio alcaligenes & 3 & 一 & - & - & 3 & $\mathbf{0}$ & $\mathbf{0}$ & $\mathbf{0}$ & 1 \\
\hline Vibrio alginolyticus & 1 & - & - & - & 1 & $\mathbf{0}$ & $\mathbf{0}$ & $\mathbf{0}$ & $\mathbf{0}$ \\
\hline Vibrio cholerae & 15 & 15 & - & - & 一 & 15 & 15 & $\mathbf{0}$ & 15 \\
\hline Vibrio eltor & 12 & 12 & - & - & - & 12 & 12 & $\mathbf{0}$ & 12 \\
\hline Vibrio metschnikovii & 1 & 1 & - & - & - & 1 & 1 & NG & 1 \\
\hline Vibrio proteus & 2 & 2 & - & 一 & - & 2 & 2 & NG & 2 \\
\hline Vibrio parahaemolyticus & 1 & 1 & - & - & - & 0 & $\mathbf{0}$ & 0 & $\mathbf{0}$ \\
\hline Vibrio spp. & 7 & 7 & - & - & - & 3 & 3 & 2 & 7 \\
\hline Yersinia enterocolitica & 7 & 7 & - & - & - & 1 & 2 & 1 & 2 \\
\hline Yersinia pestis & 12 & 12 & - & - & - & $\mathbf{0}$ & $\mathbf{0}$ & NG & 12 \\
\hline Yersinia rodentum & 23 & 23 & - & - & - & $\boldsymbol{\theta}$ & $\mathbf{0}$ & NG & 23 \\
\hline Zoogloea ramigera & 1 & - & 1 & - & - & $\mathbf{0}$ & $\mathbf{0}$ & 0 & $\mathbf{0}$ \\
\hline
\end{tabular}

Table I continued

${ }^{1}$ Ferm = fermentative; Ox = oxidative; Alk = alkali-producing; nil = no reaction. ${ }^{\text {s } N G}=$ no growth. 
ACID FROM LACTOSE TEST

Three concentrations of lactose were used in the following media.

$\begin{array}{lc}\text { Medium } A(1 \cdot 0 \% \text { w/v lactose peptone } & \text { water }) \\ \text { Peptone (Evans) } & 2 \mathrm{~g} \\ \mathrm{NaCl} & 1 \mathrm{~g} \\ \text { Lactose } & 2 \mathrm{~g} \\ 1 \% \text { w/v Bromcresol purple } & 0.4 \mathrm{ml} \\ \text { Distilled water } & 200 \mathrm{ml}\end{array}$

Medium B $(5.0 \mathrm{w} / \mathrm{v}$ lactose peptone water $)$

Peptone (Evans)

$\mathrm{NaCl}$

$2 \mathrm{~g}$

Lactose

$1 \mathrm{~g}$

Andrade's indicator

$10 \mathrm{~g}$

Distilled water

$10 \mathrm{ml}$

$190 \mathrm{ml}$

Medium $C(10 \cdot 0 \%$ w/v lactose agar slopes $)$

Lab Lemco (Oxoid)

$3 \mathbf{g}$

Peptone (Evans)

Lactose

$5 \mathrm{~g}$

Agar

$100 \mathrm{~g}$

Bromcresol purple

$10 \mathrm{~g}$

Distilled water

$0.025 \mathrm{~g}$

$1000 \mathrm{ml}$

Results in all three media were read up to 10 days' incubation at optimum temperature, usually $37^{\circ} \mathrm{C}$.

O-F TEST

The method and medium of Hugh and Leifson (1953), modified to give a final concentration of bromthymol blue of $0.006 \% \mathrm{w} / \mathrm{v}$, was used to determine the oxidative or fermentative breakdown of glucose.

\section{Results and Discussion}

The results of the O-F test were recorded as either fermentative, oxidative, alkali-producing, or nil. These results and those for the acid from lactose tests, and the ONPG test are given in table $I$. All four possible combinations of acid from lactose positive or negative and ONPG positive or negative
S. F. Lapage, Androulla Efstratiou, and L. R. Hill

were found, acid production from lactose being $\stackrel{0}{\vec{\sigma}}$ scored positive if positive by any of the three. methods. The numbers of strains in each combina- $\overrightarrow{\vec{c}}$ tion are given in table II, which also gives the numbers of strains in each $\mathrm{O}-\mathrm{F}$ reaction pattern. No discrepancies were found between our data and $\overline{\bar{\omega}}$. those of Le Minor and Ben Hamida (1962) and $\overrightarrow{\widetilde{D}}$ Bülow (1964) among those taxa studied by those $\varrho$ authors and ourselves.

\section{LACTOSE AND ONPG NEGATIVE}

The largest proportion of strains (59.5\%) were both lactose and ONPG negative and need not be discussed further except to say that they included? Brucella, Campylobacter, Comamonas, Haemophilus, $\stackrel{\circ}{\circ}$ Moraxella, Neisseria (except $N$. lactamicus), Pasteur-ella, and several Pseudomonas spp. (In this, and 0

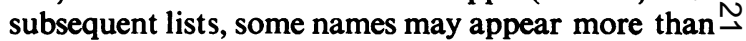
once as different strains of the same taxon behaved differently.)

\section{LACTOSE AND ONPG POSITIVE}

These comprised $15 \%$ of all the strains and were $\stackrel{\Phi}{3}$ mainly fermentative (see table II): Actinobacillus $\Phi$ spp., Aeromonas spp., Campylobacter fetus, Erwinia $\overrightarrow{\vec{O}}$ herbicola (synonym: Chromobacterium typhiflavugu, an enterobacterial species, Graham and Hodgkiss, 1967), C. lividum, Flavobacterium meningosepticusim. Gemella haemolysans, some Haemophilus sp Neisseria lactamicus, exceptional strains of Pasteurella multocida, Plesiomonas shigelloides, Pseudomonas cepacia (exceptional strains), and Yersinia enterocolitica.

\section{LACTOSE POSITIVE, ONPG NEGATIVE}

These comprised $14 \%$ of all the strains. Two were fermentative (exceptional strains of Pasteurella haemolytica), but all the remainder were oxidative: 58 strains of Pseudomonas spp, 18 strains of Acinetobacter anitratus, and five strains of Achromobacter spp. These 81 strains were all positive on the $10 \%$ lactose slopes and 32 of them also positive in the $5 \%$ lactose broths but only one positive result was obtained in the $1 \%$ lactose broths. It is interesting

\begin{tabular}{|c|c|c|c|c|c|c|}
\hline \multicolumn{2}{|c|}{ Result for } & \multicolumn{4}{|c|}{ O-F Reaction Pattern } & \multirow[t]{2}{*}{ Totals } \\
\hline Lactose & ONPG & Ferm. ${ }^{1}$ & $o x$. & Alk. & Nil. & \\
\hline+ & + & 72 & 10 & 1 & 6 & $89(15 \%)$ \\
\hline+ & - & 2 & 81 & 0 & o & $83(14 \%)$ \\
\hline$\overline{-}$ & \pm & 53 & 9 & 1 & 5 & $68(11.5 \%)$ \\
\hline \multirow{2}{*}{\multicolumn{2}{|c|}{ Totals }} & & 51 & 54 & 153 & $348(59 \cdot 5 \%)$ \\
\hline & & 217 & 151 & 56 & 164 & 588 \\
\hline
\end{tabular}

Table II Numbers of strains in the four combinations of acid from lactose and ONPG results and the four $O-F$ reaction 0 patterns.

${ }^{1}$ Ferm. = fermentative; $\mathrm{Ox}=$ oxidative; alk = alkali producing; nil = no reaction. 
to note that of the 18 Acinetobacter anitratus strains studied here, 15 were positive in the $5 \%$ lactose broths as well as on the $10 \%$ lactose slopes. Thus it would appear that in this set of 81 strains, the $5 \%$ lactose broth test, together with a negative ONPG test result, is indicative of the lactobionic acid pathway.

\section{LACTOSE NEGATIVE AND ONPG POSITIVE}

These comprised $11.5 \%$ of all the strains. They included Actinobacillus spp, Aeromonas spp, Chromobacterium typhiflavum (one strain), C. lividum Flavobacterium meningosepticum, Haemophilus paraphrophilus, Pasteurella pneumotropica, a few Pseudomonas spp (including $P$. atlantica which has a DNA base composition of $55 \% \mathrm{GC}$, atypical of the genus), Vibrio alcaligenes, Yersinia pestis, and $Y$. rodentium (synonym: Pasteurella pseudotuberculosis).

In conclusion, although the majority of the strains tested were both lactose and ONPG negative, the numbers found in the other three categories demonstrate the value of these tests together in the identification and differentiation of non-fermentative and fermentative bacterial genera in addition to those of the family Enterobacteriaceae.

The authors wish to pay tribute to the late Dr K. J. Steel for his work on an earlier, unpublished study of the same nature as this present paper.

\section{References}

Bülow, P. (1964). The ONPG test in diagnostic bacteriology. 2. Comparison of the ONPG test and the conventional lactosefermentation test. Acta path. microbiol. scand., 60, 387-402.
Costin, I. D. (1966). Results obtained by means of the beta-galactosidase test used in the routine identification of Enterobacteriaceae. Zbl. Bakt., I. Abt. Orig., 200, 49-55.

Giammanco, G. (1966). La $\beta$-D-galactosidase dans le groupe ProteusProvidencia. Ann. Inst. Pasteur, 110, 120-125.

Graham, D. C., and Hodgkiss, W. (1967). Identity of Gram-negative, yellow pigmented, fermentative bacteria isolated from plants and animals. J. appl. Bact., 30, 175-189.

Hill, L. R., Snell, J. J. S., and Lapage, S. P. (1970). Identification and characterisation of Bacteroides corrodens. J. med. Microbiol., 3, 483-491.

Hugh, R., and Leifson, E. (1953). The taxonomic significance of fermentative versus oxidative metabolism of carbohydrates by various gram negative bacteria. J. Bact., 66, 24-26.

Lapage, S. P., Hill, L. R., and Reeve, J. D. (1968). Pseudomonas stutzeri in pathological material. J. med. Microbiol., 1, 195-202.

Lapage, S. P., and Jayaraman, M. S. (1964). Beta-galactosidase and lactose fermentation in the identification of enterobacteria including salmonellae. J. clin. Path., 17, 117-121.

Le Minor, L., and Ben Hamida, F. (1962). Advantages de la recherche de la $\beta$-galactosidase sur celle de la fermentation du lactose en milieu complexe dans le diagnostic bactériologique, en particulier des Enterobacteriaceae. Ann. Inst. Pasteur, 102, 267-277.

Lowe, G. H. (1962). The rapid detection of lactose fermentation in paracolon organisms by the demonstration of $\beta$-D-galactosidase. J. med. Lab. Technol., 19, 21-25.

Lubin, A. H., and Ewing, W. H. (1964). Studies on the beta-D-galactosidase activities of Enterobacteriaceae. Publ. Hlth Lab., 22, 83-101.

Miller, J. A., and Von Riesen, V. L. (1968). Carbohydrate metabolism of Bacterium anitratum. Amer. J. med. Technol., 34, 30-34.

Snell, J. J. S., Hill, L. R., and Lapage, S. P. (1972). Identification and characterization of Moraxella phenylpyruvica. J. clin. Path., 25, 959-965.

Stodola, F. H., and Lockwood, L. B. (1947). The oxidation of lactose and maltose to bionic acids by Pseudomonas. J. biol. Chem., 171, 213-221.

Szturm-Rubinsten, S., and Piéchaud, D. (1962). Sur l'utilisation du lactose par les germes du groupe Alkalescens-Dispar. Ann. Inst. Pasteur, 103, 935-939.

Szturm-Rubinsten, S., and Piéchaud, D. (1963). Observations sur la recherche de la $\beta$-galactosidase dans le genre Shigella. Ann. Inst. Pasteur, 104, 284-291.

Tacquet, A., Tison, F., Polspoel, B., Roos, P., and Devulder, B. (1966). Etude de la $\beta$-D-galactosidase des mycobactéries. Ann. Inst. Pasteur, 111, 86-89.

Villecourt, P., and Blachère, H. (1955). Oxydation du lactose en acide lactobionique par des bactéries pathogènes ou saprophytes (B. anitratum). Ann Inst. Pasteur, 88, 523-526. 